\title{
DISPLASIA DO DESENVOLVIMENTO DO QUADRIL: ATUALIZAÇÃO
}

\author{
DYSPLASIA OF HIP DEVELOPMENT: UPDATE
}

\section{RESUMO}

A terminologia "Displasia do Desenvolvimento do Quadril DDQ" descreve o amplo espectro de alterações que atingem o quadril em crescimento, desde a displasia até a luxação da articulação, passando pelos diferentes graus de subluxação da coxofemoral. A incidência da DDQ é variável, dependendo de vários fatores, inclusive da localização geográfica. Aproximadamente um em cada 1.000 recém-nascidos poderá nascer com o quadril luxado e cerca de 10 em 1.000 com o quadril subluxado (instável). Em nosso meio podemos esperar a incidência de cinco por 1.000 quanto à positividade do sinal de Ortolani, que é o sinal clínico precoce de detecção da afecção. Os fatores de risco para a DDQ incluem: sexo feminino, raça branca, primiparidade, mãe jovem, apresentação pélvica ao nascimento, história familiar, oligohidrâmnio, recém-nascido com maiores peso e altura e com deformidades nos pés ou na coluna vertebral. O exame do quadril do recém-nascido deverá ser rotineiro e enfatizado nos berçários. No recém-nascido e nos bebês o diagnóstico da DDQ é eminentemente clínico e realizado com as manobras de Ortolani e de Barlow. A radiografia convencional tem um valor limitado na confirmação diagnóstica da DDQ nos recém-nascidos sendo a ultrassonografia do quadril o exame ideal. O tratamento da DDQ é desafiador tanto para o ortopedista pediátrico como para o generalista. Os objetivos do tratamento incluem o diagnóstico o mais precocemente possível, a redução da articulação e a estabilização do quadril em uma posição segura. Classicamente dividimos as possibilidades do tratamento de acordo com as diferentes faixas etárias, por ocasião do diagnóstico.

Descritores - Quadril/crescimento \& desenvolvimento; Luxação congênita do quadril; Doenças do desenvolvimento ósseo

\section{ABSTRACT}

The term "Developmental Dysplasia of the Hip - DDH" includes a wide spectrum of abnormalities in the immature hip, ranging range from subtle dysplasia to joint dislocation. The incidence of DDH is variable, and depends on a number of factors, such as geographical location. Approximately one in 1,000 newborn infants may present hip dislocation and 10 in 1,000 present hip instability. Brazil has an incidence of five per 1,000 in terms of the positive Ortolani signal, which is the early clinical signal for detecting the disorder. The risk factors for DDH include: female sex, Caucasian race, first labor, young mother, breech presentation on birth, family history, oligohydramnios, newborn with higher weight and height, and deformities of the feet or spine. Hip examination of the newborn should be routine, and should be emphasized in maternity units. In newborn infants and babies, the diagnosis of DDH is preeminently clinical and is carried out using the Ortolani and Barlow maneuvers. Conventional $X$-ray is of limited value for confirming the diagnosis of $\mathrm{DDH}$ in the newborn infant, and ultrasound of the hip is the ideal exam. The treatment of $\mathrm{DDH}$ is challenging, both for the pediatric orthopedist and for the general practitioner. The objectives of the treatment include early diagnosis, reduction of the articulation, and stabilization of the hip in a secure position. Classically, treatment options are divided according to the different age groups, at the time of diagnosis.

Keywords - Hip/growth \& development; Hip dislocation, congenital hip deformity; Developmental bone disease

Professor Associado Livre Docente do Departamento de Ortopedia e Traumatologia da Faculdade de Medicina da Universidade de São Paulo.

Correspondência: Rua Ovídio Pires de Campos, 333 - 3oandar - Cerqueira César - São Paulo, SP - E-mail: robertoguarniero@clinicareboucas.com.br 


\section{INTRODUÇÃO}

Estamos adotando e propondo a divulgação da terminologia "Displasia do Desenvolvimento do Quadril - DDQ” em substituição à denominação anterior "Luxação Congênita do Quadril - LCQ”, pois esta nova nomenclatura descreve com maior precisão o espectro de alterações possíveis na afecção do quadril do recémnascido. DDQ é um termo genérico que descreve uma ampla faixa de anormalidades anatômicas do quadril, quer de natureza congênita, ou desenvolvidas nos primeiros meses de vida da criança.

Na realidade, a terminologia DDQ descreve o amplo espectro de alterações que atingem o quadril em crescimento, desde a displasia até à luxação da articulação, passando pelos diferentes graus de subluxação da coxofemoral. Esta nova denominação é endossada pela AAOS - American Academy of Orthopaedic Surgeons, pela AAP - American Academy of Pediatrics, pela POSNA - Pediatric Orthopaedics Society of North America, pela EPOS - European Pediatric Orthopaedics Society e pela SBOP - Sociedade Brasileira de Ortopedia Pediátrica ${ }^{(1,2)}$.

\section{DEFINIÇÕES}

Displasia do quadril é um termo que denota uma anormalidade no tamanho, na morfologia, na orientação anatômica ou na organização da cabeça femoral, na cavidade acetabular ou em ambos. A displasia acetabular é caracterizada pelo acetábulo imaturo, com a cavidade rasa que pode acarretar a subluxação ou a luxação da cabeça femoral.

Na subluxação do quadril, a cabeça femoral está deslocada de sua posição anatômica normal, mas ainda mantém algum contato com a cavidade acetabular. Na luxação do quadril não ocorre nenhum contato entre a cabeça femoral e a cavidade acetabular.

Denominamos “quadril instável” aquela articulação que se apresenta reduzida, na posição anatômica, mas em que é possível provocarmos a subluxação, ou a luxação da articulação.

Luxação teratológica - É produzida nos primeiros meses da vida intrauterina e ao nascimento reconheceremos não só a luxação como existirão alterações morfológicas de tal grau que a redução da articulação será muito difícil, senão impossível. Aqui incluem-se as luxações associadas à artrogripose, à síndrome de Larsen e à deficiência femoral proximal (com todas as suas variantes), as neuromusculares e as que ocorrem nas síndromes genéticas. Neste grupo praticamente só encontramos quadris luxados, sendo inexistentes os quadros de subluxação ou de instabilidade. Não serão discutidos neste artigo.

\section{EPIDEMIOLOGIA E ETIOLOGIA}

A incidência da DDQ é variável, dependendo de vários fatores, inclusive da localização geográfica. Aproximadamente um em cada 1.000 recém-nascidos poderá nascer com o quadril luxado e cerca de 10 em 1.000 com o quadril subluxado (instável).

Em nosso meio podemos esperar a incidência de, aproximadamente, cinco por 1.000 quanto à positividade do sinal de Ortolani, que é o sinal clínico precoce de detecção da afecção, como será posteriormente estudado $^{(3,4)}$.

Os fatores de risco para a DDQ incluem: sexo feminino, raça branca, primiparidade, mãe jovem, apresentação pélvica ao nascimento, história familiar, oligohidrâmnio, recém-nascido com maiores peso e altura e com deformidades nos pés ou na coluna vertebral.

O quadril esquerdo é o mais afetado (60\%) e o quadril direito é o menos afetado (20\%) nas situações de unilateralidade, sendo a bilateralidade menos frequente (20\%).

Por razões desconhecidas, a DDQ afeta em menor frequência os indivíduos da raça negra.

Existe um acometimento maior nos italianos e descendentes, inclusive quando estudamos as populações que emigraram para outras regiões geográficas ${ }^{(5)}$.

\section{EXAME FÍsICO}

A situação ideal é o exame no berçário, ou nos primeiros dias de vida. Obviamente caracterizamos a história, os fatores de risco e os antecedentes, como anteriormente mencionado, para a elaboração do diagnóstico.

Os métodos para o diagnóstico precoce da DDQ são descritos na literatura há pelo menos 50 anos. Na Itália, Putti iniciou um programa pioneiro de diagnóstico precoce e tratamento da afeç̧ão e publicou seus resultados em $1926^{(6)}$. Nos EUA, Howorth, em 1932, realizou o trabalho pioneiro de diagnóstico precoce da afecção, no "Babies Hospital” de Nova York. Outros autores aparecem, então, na literatura, mas foi na década de 40, após a 2 ${ }^{\underline{a}}$ Guerra Mundial, que os trabalhos com maior abrangência de crianças são descritos nos Estados Unidos, Suécia e Inglaterra ${ }^{(5,7)}$. 
O diagnóstico precoce pode ser considerado como relativamente simples, seguro e que proporciona um tratamento geralmente eficaz. Resultados obtidos com o diagnóstico precoce e classificados como bons e excelentes são referidos tanto na área ortopédica como na área pediátrica ${ }^{(5,7)}$. O tratamento precoce proporciona níveis de, aproximadamente, $96 \%$ bons resultados.

O exame do quadril do recém-nascido deverá, portanto, ser rotineiro e enfatizado ainda no berçário e, também, no seguimento ambulatorial da criança, nas primeiras semanas e meses de vida.

No recém-nascido e nos bebês o diagnóstico da DDQ é eminentemente clínico e realizado com as manobras de Ortolani e de Barlow. Estes testes tomarão muito pouco tempo na rotina do exame físico do recémnascido ${ }^{(8-11)}$.

O sinal de Ortolani é um teste de redução do quadril, ou seja, quando um recém-nascido com a articulação coxofemoral luxada é examinado, a cabeça femoral é reduzida no acetábulo com a manobra, assim sendo demonstrada a luxação. A manobra é realizada com a criança em decúbito dorsal com os quadris e os joelhos em posição de flexão de $90^{\circ}$, com as coxas em adução e com rotação interna leves. Examinamos um dos quadris de cada vez, com o outro bem estabilizado, em posição de leve abdução. Ao efetuarmos um movimento de abdução dos quadris, podendo ser acompanhado por uma leve rotação externa das coxas, poderemos ter a sensação de um "ressalto" na articulação patológica (às vezes poderemos ter até uma sensação audível desse “ressalto”). O sinal é dito, então, Ortolani positivo (Figura 1).

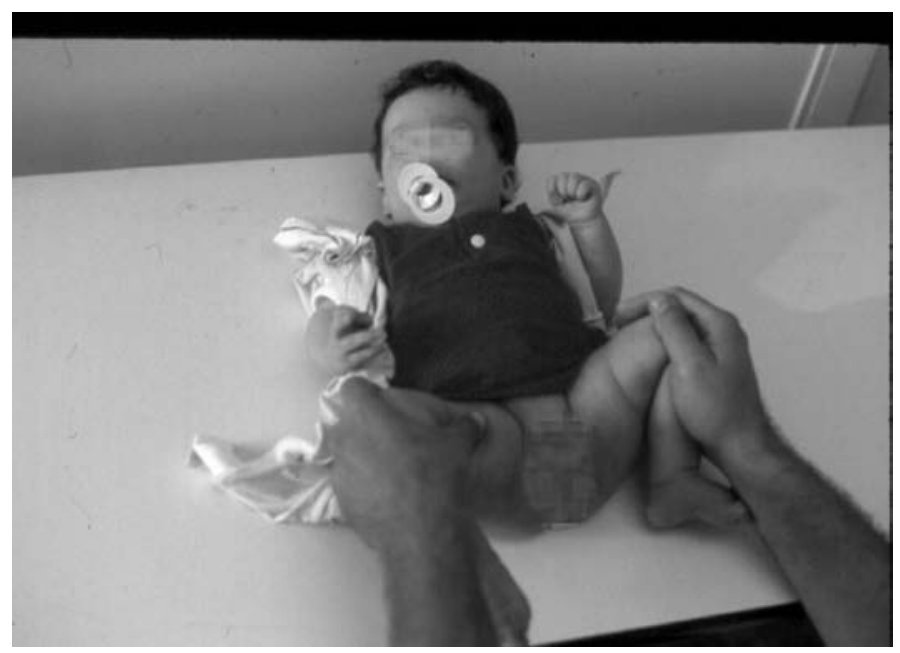

Figura 1 - Fotografia ilustrativa da forma correta de realizar a manobra de Ortolani
O sinal de Barlow é exatamente o oposto, ou seja, é uma manobra provocativa da luxação de um quadril instável. O teste de Barlow determinará o potencial para a luxação do quadril examinado. A coxa do paciente é mantida em ângulo reto com o tronco, em posição de adução; força será exercida pelo joelho da criança em direção vertical ao quadril, em um esforço para deslocar a cabeça femoral do interior do acetábulo. O examinador procura um sinal de "pistonagem" no quadril em exame, que poderá ou não ser acompanhado por "ressalto”. Se o quadril é reduzido pela manobra de Ortolani, a cabeça femoral ficará perfeitamente alojada na cavidade acetabular; entretanto, com a flexão do quadril e levando-se a coxa para a posição de adução, a cabeça femoral luxa, podendo ser deslocada posteriormente em relação ao acetábulo, caracterizando, então, o quadril instável. No teste de Barlow, a parte superior do fêmur é mantida entre os dedos indicador e médio e sobre o trocanter maior, e o polegar na região inguinal. A cabeça femoral poderá ser alavancada para dentro e para fora da articulação, confirmando a instabilidade.

A manobra de Ortolani não deverá ser executada com esforço e, sim, com delicadeza e com a criança perfeitamente calma, pois é uma redução do quadril luxado. Uma criança que está chorando muito, agitada, dificultará e atrapalhará sobremaneira a realização e a conclusão do exame.

Em poucas semanas, se o quadril permanecer luxado, a limitação da abdução da articulação será evidente e um sinal clinico importante. A abdução máxima dos quadris do recém-nascido, considerada como normal, tem um valor acima dos 60 graus.

Com o crescimento da criança e o quadril luxado, os sinais clínicos tornam-se mais óbvios. Com o passar do tempo, torna-se mais difícil e impossível a redução da luxação pela manobra de Ortolani, passando, então, este sinal a ser negativo. A limitação da abdução tornase maior.

Entre os três e os seis meses de idade, a redução do quadril de uma criança acordada poderá ser difícil; por isto, nesta faixa etária não é comum encontrarmos crianças com o sinal de Ortolani positivo. Portanto, neste grupo, a manobra de Ortolani perde o seu valor diagnóstico.

O examinador deve considerar, também, o fato de a criança apresentar "estalidos" quando da realização do exame físico que podem não ser do quadril instável ou luxado. 
Os métodos de exame do quadril no recém-nascido são mostrados aos estudantes de medicina ou aos médicos residentes com material audiovisual, ou utilizando-se os bebês no berçário. A necessária experiência no exame clínico, fundamental para o médico pediatra, berçarista ou neonatologista, só será adquirida quando examinados os recém-nascidos com a afecção. Estas dificuldades são superadas com a utilização de um modelo para o ensino e o treinamento dos estudantes e demais profissionais da saúde ${ }^{(12,13)}$ (Figura 2).

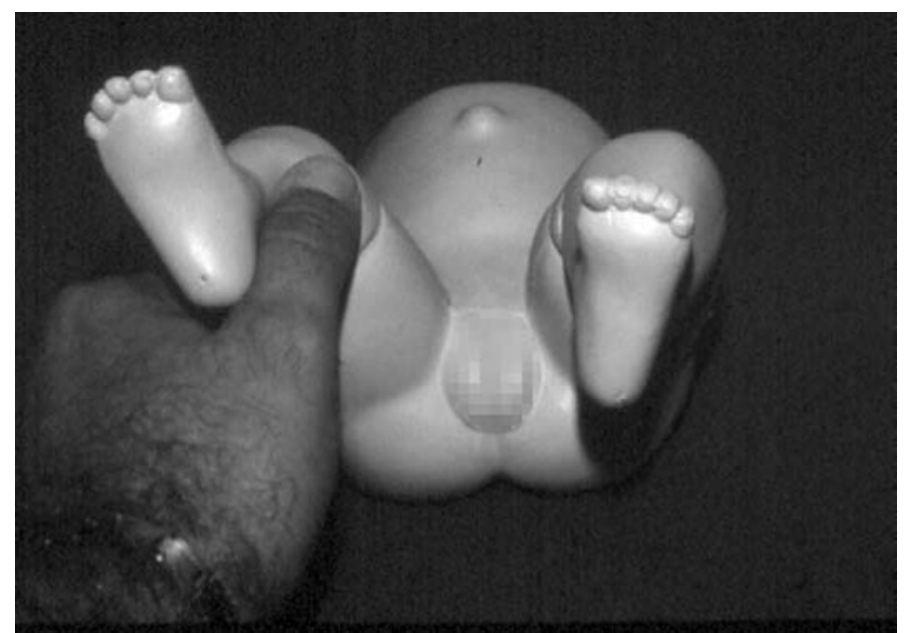

Figura 2 - Modelo de ensino ilustrando a manobra de Ortolani

Um outro sinal tardio é o de Galeazzi ou de Allis: com a criança deitada e com os joelhos fletidos, eles não estarão na mesma altura, denotando já a diferença de comprimento entre os membros inferiores. Evidentemente este sinal será mais claro nos casos unilaterais (Figura 3).

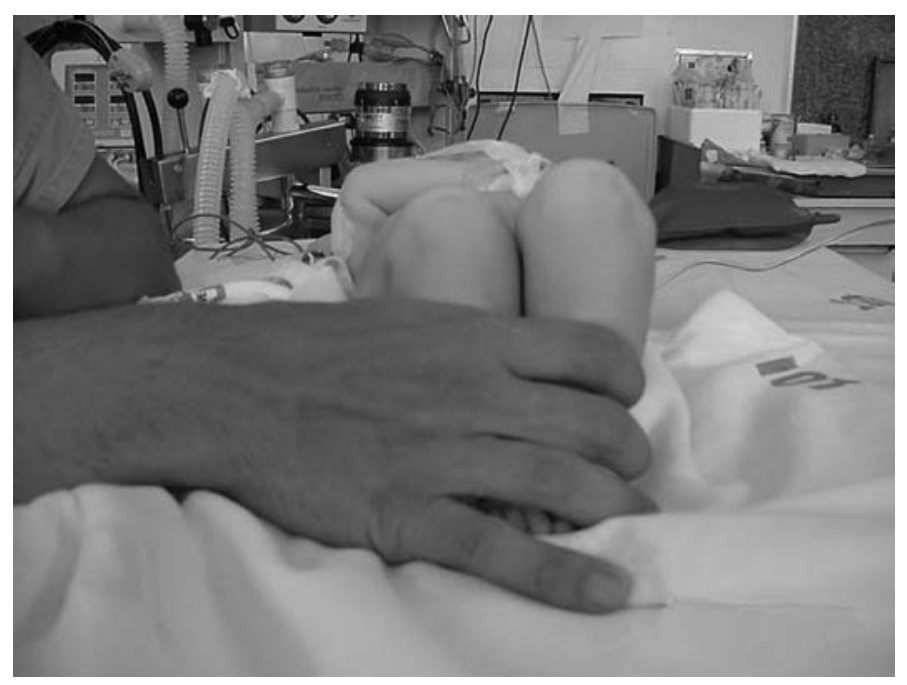

Figura 3 - Fotografia ilustrativa do sinal de Galeazzi
Pode ocorrer, também, assimetria nas pregas glúteas cutâneas, mas isto não é uma constante.

De qualquer forma, acreditamos que o médico berçarista, ou o neonatologista, deverá enviar a criança para consulta especializada com o ortopedista nas seguintes eventualidades:

1) achados clínicos de instabilidade ou de luxação do quadril;

2) nos casos de dúvida; e,

3) os pacientes de "alto risco" - parto pélvico, primeira gestação, mãe jovem, antecedentes familiares - independentemente do resultado do exame físico efetuado.

\section{EXAMES DE IMAGEM}

A radiografia convencional tem um valor limitado na confirmação diagnóstica da DDQ nos recém-nascidos, pois as epífises femorais proximais (cabeças dos fêmures) não estão ossificadas até os quatro a seis meses de vida. Por outro lado, a ultrassonografia do quadril do neonato tem um potencial óbvio nas crianças desta faixa etária, pois evidenciará claramente as estruturas cartilaginosas que são precariamente delineadas pela radiografia simples ${ }^{(14-16)}$.

A radiografia da bacia começará a ser útil para a confirmação da DDQ mais tarde, após os quatro meses de idade da criança. Ressaltamos que o núcleo de ossificação da cabeça femoral será radiograficamente visível a partir do quarto ou sexto mês de vida.

Na avaliação radiográfica para o diagnóstico da DDQ, nos primeiros meses de vida, temos que lançar mão de medidas e sinais indiretos, tais como: linhas dos quadrantes, linha horizontal de Hilgenreiner e linha vertical de Perkins, linha de Shenton e o índice acetabular (Figura 4).

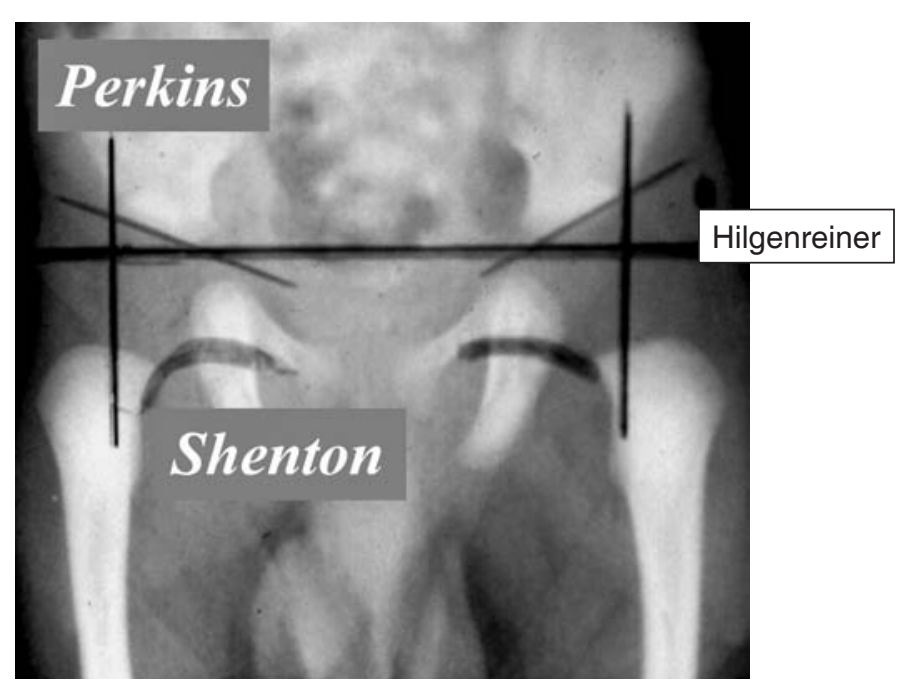

Figura 4 - Linhas na radiografia da bacia 
A tomografia axial computadorizada (TAC) no estudo da DDQ, graças ao seu poder de resolução e à possibilidade de realizar os cortes transversais, permite a observação da interposição do tendão do músculo ileopsoas e da hipertrofia do pulvinar, mesmo sem a contrastação da cápsula e do interior da cavidade articular como, também, evidenciar as subluxações femorais e a medida do ângulo de anteversão acetabular. Este exame poderá ser realizado mesmo com a criança usando aparelho gessado e será útil, portanto, para informar a exata posição de redução. Em trabalho realizado no Hospital das Clínicas da Faculdade de Medicina da Universidade de São Paulo e publicado em 1990, foram estudados nove pacientes com 11 quadris luxados submetidos à redução incruenta e à imobilização em aparelhos gessados e feito o controle desta redução com a TAC. A conclusão foi de que a TAC é um bom método na avaliação da redução incruenta da DDQ, sendo útil na maioria dos casos em que a radiografia simples deixar dúvidas; o ângulo de anteversão acetabular nem sempre está aumentado na DDQ como foi comprovado pela medição realizada no trabalho; e, o pulvinar hipertrofiado pode muito bem ser detectado pela $\mathrm{TAC}^{(17)}$.

\section{TRATAMENTO}

O tratamento da DDQ é desafiador tanto para o ortopedista pediátrico como para o generalista. Os objetivos do tratamento incluem o diagnóstico o mais precocemente possível, a redução da articulação e a estabilização do quadril em uma posição segura.

Classicamente, dividimos as possibilidades do tratamento de acordo com as diferentes faixas etárias, por ocasião do diagnóstico.

\section{a) Tratamento do recém-nascido até aos três meses de vida}

O tratamento é indicado tão cedo o diagnóstico tenha sido realizado. Nesta faixa etária o tratamento é baseado no conceito de que, mantendo-se o posicionamento do quadril reduzido em flexão e em leve abdução, ocorrerá o estímulo necessário para o desenvolvimento normal da articulação. Assim, uma vez estabelecido o diagnóstico de instabilidade ou de luxação do quadril, o tratamento será iniciado visando a redução da cabeça femoral na cavidade acetabular e a sua manutenção até a certeza da estabilidade articular.

Inúmeros aparelhos ortopédicos estão disponíveis nos dias de hoje para cumprir o objetivo do tratamento inicial. Atualmente, a órtese mais comumente usada é o suspensório de Pavlik (Figura 5). Esta órtese proporciona a simul- tânea flexão e abdução da articulação coxofemoral graças às tiras que se unem com relativa facilidade. Segundo Pavlik ${ }^{(18)}$, o uso do suspensório por ele idealizado diminui o risco da necessidade de redução cirúrgica.
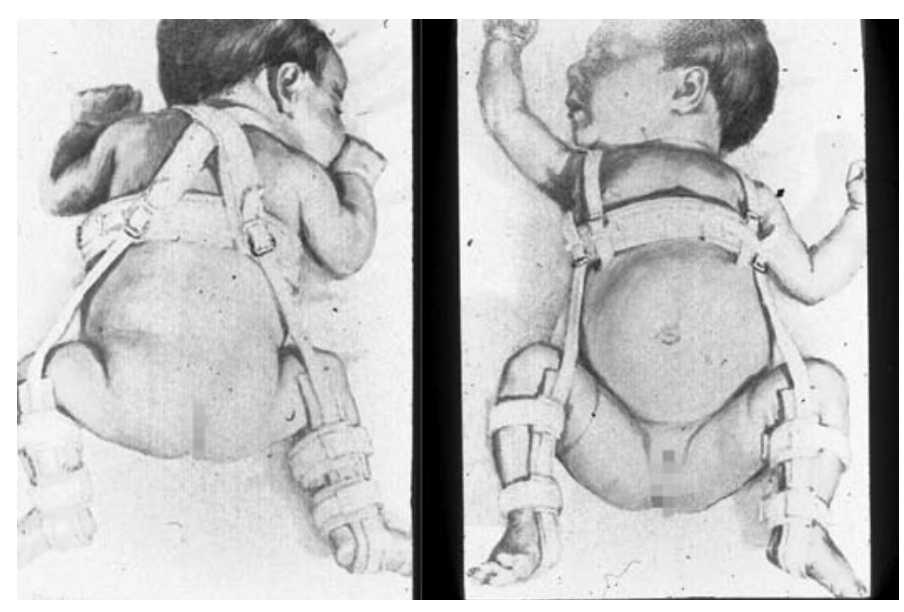

Figura 5 - Fotografia ilustrativa do suspensório de Pavlik

Ramsey et al $^{(19)}$ descrevem a forma apropriada para o uso do suspensório de Pavlik, cuja ação está baseada no princípio de redução em flexão, evitando uma posição de abdução forçada da articulação. No trabalho, os autores mostram que $89 \%$ dos quadris luxados em crianças menores de seis meses de idade foram reduzidos com sucesso e apresentaram um desenvolvimento normal com o uso desta órtese; apenas um quadril apresentou alterações leves, tipo osteocondrite. Em dois pacientes a falha na obtenção da redução foi devido ao não posicionamento em flexão de $90^{\circ}$. Os autores recomendam o uso do suspensório de Pavlik no período de recémnascido até aos nove meses de idade não referindo necrose avascular da epífise femoral, o que está de acordo com autores como Kalamchi e MacFarlane ${ }^{(20)}$. Porém, se a redução concêntrica não é obtida nas primeiras duas a três semanas de uso do suspensório, esta conduta será abandonada em favor da tração seguida pelas técnicas clássicas de redução do quadril.

As falhas de redução com o uso do Pavlik geralmente são devidas a um mau acompanhamento da criança pelo médico no ambulatório. Quando a opção é pelo uso do suspensório, é necessário que a criança seja examinada com frequência para avaliar a correta aplicação do mesmo, geralmente, a cada semana. Em linhas gerais, a criança permanecerá seis a oito semanas usando o aparelho ou, como regra prática, aproximadamente duas vezes o valor da idade em que iniciou o uso do suspensório de Pavlik. Lembramos que o suspensório de Pavlik poderá ser usado até aos quatro ou seis meses de idade da criança ${ }^{(21)}$. 
Em caso de falha com o uso do suspensório a nossa opção é a redução incruenta e a imobilização em aparelho gessado (precedida ou não por um período de tração) para esta faixa etária.

\section{b) Tratamento dos três meses de vida até a idade da marcha}

Nesta faixa etária a maioria dos pacientes com DDQ poderá ser tratada com a redução incruenta (fechada) e a imobilização em aparelho gessado pelvipodálico ${ }^{(22)}$. No ato operatório poderá ser necessária a tenotomia percutânea dos músculos adutores do quadril.

Quando não conseguimos a redução por manobras fechadas, a redução aberta (cruenta) está indicada. Assim, as indicações para a redução cruenta são:

1) a cabeça femoral permanece acima da cartilagem trirradiada no exame radiográfico;

2) arco de redução/luxação é menor do que $25^{\circ}$ após a tenotomia dos adutores;

3) a cabeça femoral não entra no acetábulo;

4) a cabeça femoral permanece lateralizada em relação ao acetábulo após quatro semanas de redução parcial; e

5) a redução previamente tentada falhou.

Após o período de imobilização no aparelho gessado, que variará de dois a três meses, o paciente passa a utilizar uma órtese de abdução (por exemplo, tipo Milgram) por mais dois a três meses.

\section{REFERÊNCIAS}

1. Guille JT, Pizzutillo PD, MacEwen GD. Development dysplasia of the hip from birth to six months. J Am Acad Orthop Surg. 2000;8(4):232-42.

2. Guarniero R, Lage LAA, Luzo CAM, Romano D. Complicações no tratamento da displasia do desenvolvimento do quadril (DDQ): revisão da literatura e princípios de tratamento. Acta Ortop Bras. 1994;2(1):47-9.

3. Guarniero R, Montenegro NB, Vieira PB, Peixinho M. Sinal de Ortolani: resultado do exame ortopédico em 9171 recém-nascidos na Associação Maternidade de São Paulo. Rev Bras Ortop. 1988;23(5):125-8.

4. Guarniero R. Congenital dislocation of the hip. Epidemiology. Mapfre Med. 1992;3(Suppl 2):11-3.

5. Bowen JR, Kotzias-Neto A. Developmental dysplasia of the hip. Brooklandville: Data Trace; 2006.

6. Putti V. Congenital dislocation of the hip. Surg Gynecol Obstet. 1926;42:449.

7. Howorth B. Development of present knowledge of congenital displacement of the hip. Clin Orthop Relat Res. 1977;(125):68-87.

8. Staheli LT. Ortopedia pediátrica na prática. 2 a. Porto Alegre: Artmed; 2008.

9. Skaggs DL, Flynn JM. Staying out of trouble in pediatric orthopaedics. Philadelphia: Lippincott Williams \& Wilkins; 2006.

10. Storer SK, Skaggs DL. Developmental dysplasia of the hip. Am Fam Physician. 2006;74(8):1310-6.

11. Maxwell SL, Ruiz AL, Lappin KJ, Cosgrove AP. Clinical screening for developmental dysplasia of the hip in Northern Ireland. BMJ. 2002;27;324(7344): 1031-3.

12. Guarniero R, Peixinho M, Montenegro NB. Avaliação do uso de um modelo de ensino e treinamento para o diagnóstico precoce da luxação congênita do quadril. Rev Bras Ortop. 1988;23(5):133-5.
A criança com DDQ requer avaliação clínica e radiográfica, com tratamento ortopédico e observação quando indicado, até a maturidade esquelética.

\section{c) Tratamento após a idade da marcha}

Esta necessidade não deveria existir, pois o ideal é que o diagnóstico seja efetuado bem antes desta faixa etária; entretanto, em algumas crianças, pode ocorrer a falha tanto do diagnóstico como do consequente tratamento precoce.

A abordagem e os tipos de tratamento nas crianças com idades superiores a um ano e meio ou dois anos, são motivo de controvérsia.

Pode ser indicada a tentativa de redução fechada (incruenta) ou, então, a redução aberta (cruenta) será praticamente obrigatória. Nesta faixa etária, quando do ato operatório, ainda teremos de considerar o encurtamento femoral (ósseo) para permitir a redução articular como, também, as operações com osteotomias complementares na região acetabular (osteotomias tipos Salter, Dega e outras) $)^{(23)}$.

O limite de idade para indicação das tentativas de redução do quadril será até os quatro ou cinco anos de vida da criança. Após esta idade, as denominadas operações “de salvamento" da articulação são utilizadas, incluindo as osteotomias pélvicas mais elaboradas e difíceis (tipos Steel, de Chiari, poligonal pélvica e outras) ou pensaremos nas artroplastias totais do quadril.

13. Cole WG. Evaluation of a teaching model for the early diagnosis of congenital dislocation of the hip. J Pediatr Orthop. 1983;3(2):223-6.

14. Sernik R. Ultrassonografia do sistema musculoesqueletico. Rio de Janeiro: Revinter; 2009.

15. Synder M, Harcke HT, Domzalski M. Role of ultrasound in the diagnosis and management of developmental dysplasia of the hip: an international perspective. Orthop Clin North Am. 2006;37(2):141-7.

16. Guarniero R, Grigoletto Júnior W, Barros JC, Stump XM, Homsi C. Ultrasound in the early diagnosis of congenital hip dislocation. Rev Hosp Clin Fac Med Sao Paulo. 1986;41(4):194-7.

17. Lage LAA, Rodrigues LEA, Stump XMGRG, Guarniero R, Peixinho M. A tomografia axial computadorizada na luxação congênita do quadril. Rev Bras Ortop. 1990;25(8):282-6.

18. Pavlik A. The functional method of treatment using a harness with stirrups as the primary method of conservative therapy for infants with congenital dislocation of the hip. 1957. Clin Orthop Relat Res. 1992;(281):4-10.

19. Ramsey PL, Lasser S, MacEwen GD. Congenital dislocation of the hip. Use of the Pavlik harness in the child during the first six months of life. J Bone Joint Surg Am. 1976;58(7):1000-4.

20. Kalamchi A, MacFarlane R 3rd. The Pavlik harness: results in patients over three months of age. J Pediatr Orthop. 1982;2(1):3-8.

21. Kalamchi A, MacEwen GD. Avascular necrosis following treatment of congenital dislocation of the hip. J Bone Joint Surg Am. 1980;62(6):876-88.

22. Vitale MG, Skaggs DL. Developmental dysplasia of the hip from six months to four years of age. J Am Acad Orthop Surg. 2001;9(6):401-11.

23. Forlin E, Munhoz da Cunha LA, Figueiredo DC. Treatment of developmental dysplasia of the hip after walking age with open reduction, femoral shortening, and acetabular osteotomy. Orthop Clin North Am. 2006;37(2):149-60, 